\title{
Non-traumatic ulnar artery aneurysm in a middle-aged male
}

\author{
Nalaka Gunawansa, Prasath Subramaniyam \\ National Institute of Nephrology and Transplantation, Colombo, Sri Lanka
}

\begin{abstract}
Aneurysms in the extremity arteries are rare and usually occur as pseudoaneurysms secondary to trauma or iatrogenic interventions. True spontaneous non-traumatic aneurysms in the extremities are extremely rare with no consensus on their aetiology or optimum management strategy. Here, we discuss a case of a young man with an isolated spontaneous non-traumatic ulnar artery aneurysm presenting with acute pain and discomfort.
\end{abstract}

Key words: aneurysm, ulnar artery aneurysm, Marfan syndrome, pseudoaneurysm

Acta Angiol 2020; 26, 4: 147-149

\section{Introduction}

Upper extremity arterial aneurysms (UEAA) are rare in clinical practice. Even when encountered, they are primarily pseudoaneurysms often following direct trauma or iatrogenic interventions such as arterial catheterization. True spontaneous aneurysms in the upper extremities are very uncommon and offer unique challenges in their management. While spontaneous aneurysms in the brachial artery have been reported sporadically, aneurysms arising in the forearm arteries distal to the brachial artery bifurcation are extremely uncommon [I]. Santis and colleagues (20I3) in a systematic review covering 20 years (I990-20 I I), reported just 28 cases of non-traumatic forearm aneurysms, the majority of which were distal palmar aneurysms [2]. We report a rare case of a non-traumatic ulnar artery aneurysm in a 39-year-old active male who presented with acute pain and tenderness in the forearm.

\section{Case report}

A thirty-nine-year-old male presented to the Emergency Department with sudden onset pain and swelling of the right forearm, with gradual worsening of symptoms over a 7-day period. He denied any history of direct trauma to the region preceding the onset of pain. On clinical examination, he had a tender swelling in the proximal $1 / 3$ of the right forearm with no clinical evidence of cellulitis or compartment syndrome. There was a mild ecchymotic patch over the proximal forearm. The proximal brachial artery pulse at the cubital fossa and distal radial and ulnar pulses at the wrist were present and clinically equivalent to the left side. Allen test of the right hand confirmed radial artery dominance. He had no major co-morbidities other than being on regular steroidal inhalers for chronic obstructive airway disease. He had no history of substance abuse or self-injection. He was quite active and independent, being employed as a computer programmer.

The blood biochemistry results were unremarkable with no evidence of infection or inflammation. An initial ultra-sound scan of the forearm suggested a muscular haematoma. An arterial duplex ultra-sound (DUS) assessment of the forearm revealed a possible aneurysmal dilatation of the proximal ulnar artery with normal distal arterial flow pattern. Abdominal, lower limb and carotid duplex imaging did not reveal any further aneurysmal change elsewhere. An echocardiogram did not reveal any cardiac valvular or intra-mural pathology. A computerized tomographic angiogram (CTA) was done to further visualize the region of interest.

The CTA confirmed an elongated aneurysmal segment of the proximal ulnar artery with no intramural

Address for correspondence: Nalaka Gunawansa, National Institute of Nephrology and Transplantation, Jayantha Weerasekera Mw., 00700 Colombo, Sri Lanka, e-mail: vascular@drnalakagunawansa.com 


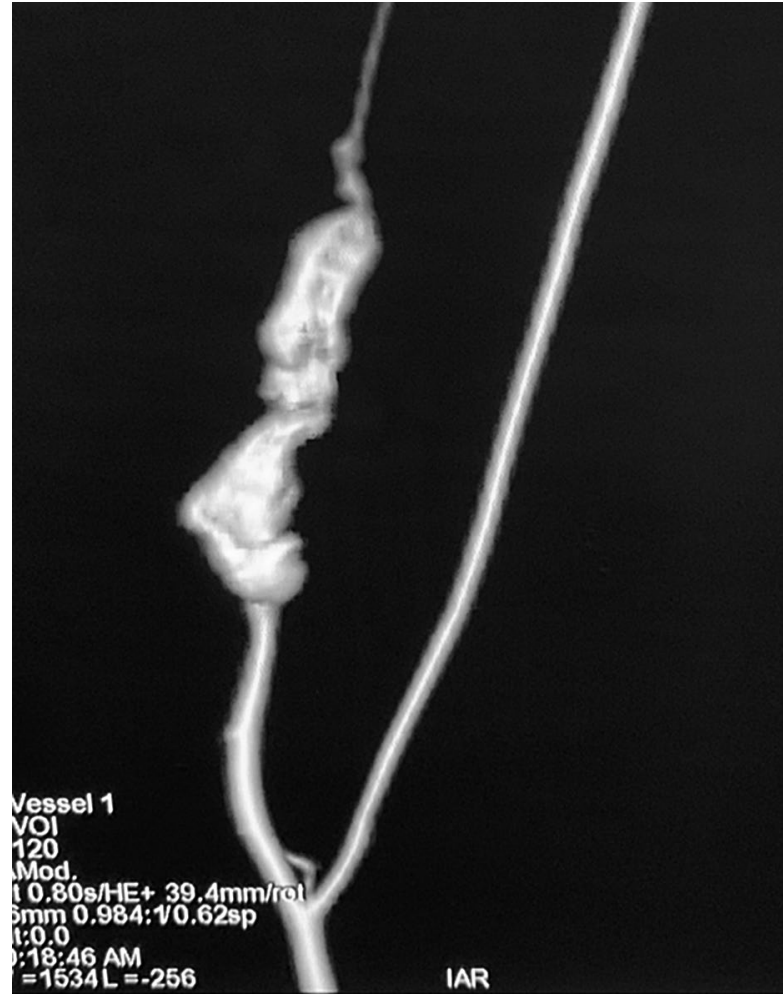

Figure I. Reconstructed CTA image showing the elongated aneurysmal segment in the proximal ulnar artery

thrombi (Figs I, 2). There was no venous filling to indicate any possibility of arterio-venous malformation. The remaining arterial architecture appeared pristine. There was no extravasation to suggest rupture.

The images were studies with regards to a possible radiological intervention with insertion of a covered stent. However, technical limitations and lack of local expertise in stenting the ulnar artery were prohibitive in the management planning. Hence, given the progressively worsening symptoms and the extent of the lesion, a decision was taken to proceed with surgical exploration.

The aneurysmal region was approached with a liner incision extending from the cubital fossa to mid forearm under a regional nerve block. Proximal and distal control was achieved by atraumatic vascular tapes. During exploration, the proximal ulnar artery was found to be extremely friable and not amenable to an arterial anastomosis or reconstruction. The ulnar artery was ligated proximal and distal to the diseased segment and the aneurysmal component was excised. The segment was sent for histological confirmation and tissue culture while the wound was closed over a vacuum drain.

The recovery from surgery was uneventful. The swelling and pain subsided rapidly, and his distal palmar and digital perfusion remained excellent. Currently,

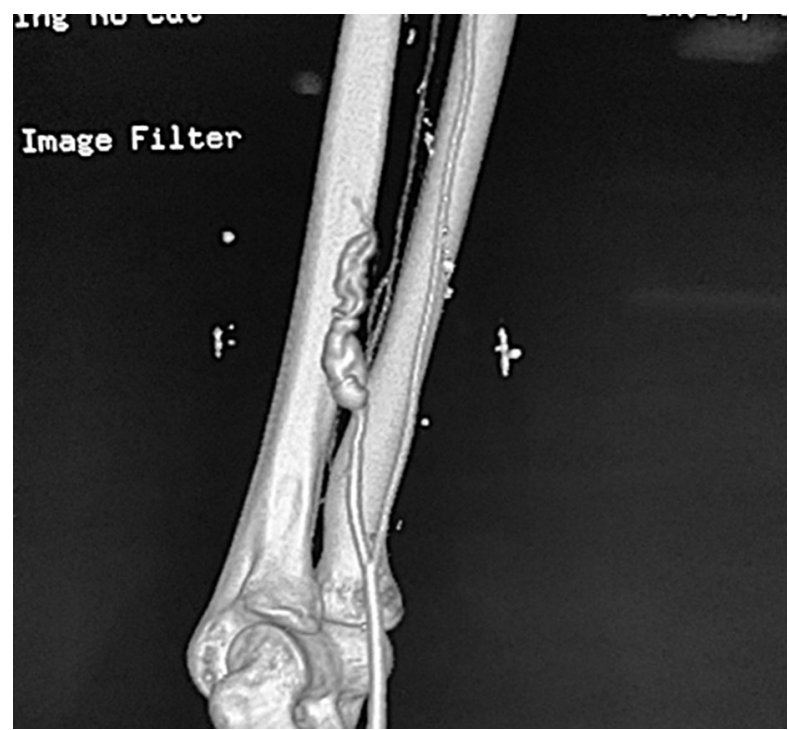

Figure 2. CT Angiogram with bone window

9 months after surgery, he remains well without symptoms, fully active and back at his usual employment. The resected segment of aneurysmal artery was examined by histology and confirmed a true aneurysm with thinned out arterial wall and no evidence of infection or inflammation. There was no evidence of atheromatous deposits, fibrinoid necrosis or arteritis. The related specimens sent for bacterial and tuberculous culture were negative. A repeat DUS examination of all extremities and neck vessels showed no similar pathology or local recurrence.

\section{Discussion}

\section{Background}

UEAA are rather uncommon compared to abdominal, visceral or lower limb artery aneurysms. Even when they do occur, it is usually secondary to repetitive trauma or direct injury resulting in pseudoaneurysms. Distal radial and ulnar artery aneurysms have been reported due to repetitive trauma to the hand among manual labourers [3, 4]. More recently with increasing incidence of endovascular interventions through radial artery approach, iatrogenic pseudoaneurysms of the distal radial artery have also been reported. Proximal forearm non-traumatic aneurysms are extremely rare and reported only as case reports in sporadic publications $[\mathrm{I}, 5]$.

\section{Diagnosis}

Accurate diagnosis requires imaging confirmation. This patient was initially evaluated with a DUS. In similar situations, DUS is the first investigation of choice. It is 
non-invasive, readily available and can demonstrate as well as localise the aneurysm. However, DUS remains highly operator dependent and may not be accurate for finer details in terms of size, extent and associated minor pathologies in the adjacent arterial tree. CTA or magnetic resonance angiogram (MRA) would be required as the gold standard imaging modality to confirm the diagnosis. Compared to CTA, MRA is more sensitive and specific for diagnosing $A V$ malformations whereas in UEAA, MRA does not provide any significant advantage over CTA [6].

\section{Aetiology}

One of the reported aetiologies of non-traumatic peripheral aneurysms is infection, leading to mycotic aneurysms. In this index patient, inflammatory markers were normal and repeated blood cultures were negative. The tissue culture taken from the excised artery was also negative for standard bacterial survey and mycobacteria. Another possible rare aetiology is primary vasculitis or auto-immune disease affecting the arterial wall. This too was unlikely in the index patient who was found negative for the complete auto-immune survey, had normal level inflammatory markers and lacked any suggestive finding on histology.

\section{Treatment}

Even in the current endovascular era there is no consensus on the ideal management of UEAA. Given the uncertain aetiology as well as complex morphological pattern of the aneurysmal segment, endovascular stenting was not possible in this patient. There are reported cases of pseudoaneurysms treated with covered stent and coil embolization [7]. We did not consider coil embolization due to the absence of a clear aneurysm neck and possibility of distal embolization in the dominant arm. Open surgical exploration gives an opportunity to excise the entire diseased segment and evaluate for histological confirmation [8]. Furthermore, if carefully performed, it allows immediate decompression of the forearm and hence relief of symptoms in addition to the definitive treatment of the aneurysm. Surgical options include complete ligation or end-to-end repair using a venous segment. While end-to-end reconstruction is preferred in the absence of any obvious infection, complete ligation is also a viable option where the distal perfusion can be reliably sustained.

\section{Conclusion}

True aneurysms of the proximal forearm arteries are very rare. Accurate diagnosis requires imaging by either CTA or MRA. Due to the uncertain nature of their natural history and associated symptomatology, definitive treatment is often required either by endovascular stenting, embolization or open surgical repair. The actual treatment modality depends on anatomy, morphological nature, availability of stents and expertise as well as adequacy of collateral distal perfusion. If further investigations confirm infective or inflammatory aetiology, long-term treatment of such primary pathology is required to prevent recurrence or occurrence of new disease elsewhere.

\section{Conflicts of interest}

None.

\section{Consent}

Specific informed written consent of the index patient was obtained for publication of above details and images for the purpose of medical education.

\section{References:}

I. Clark ET, Mass DP, Bassiouny HS, et al. True aneurysmal disease in the hand and upper extremity. Ann Vasc Surg. 1991; 5(3): 276-281, doi: 10.1007/BF02329386, indexed in Pubmed: 2064922.

2. De Santis F, Martini G, Mani G, et al. Forearm and hand arteries' aneurysms - a case report of bilateral true ulnar artery aneurysm in the hypothenar eminence and systematic review of the literature. Vascular. 2013; 21 (3): 169-176, doi: 10.1 177// 708538 | |3478732, indexed in Pubmed: 23493282.

3. Johnston K, Rutherford R, Tilson M, et al. Suggested standards for reporting on arterial aneurysms. Journal of Vascular Surgery. 1991; 13(3): 452-458, doi: 10.1067/mva. 1991.26737.

4. Kubo N, Murase T, Moritomo H, et al. Giant aneurysm of the ulnar artery in the palm treated by resection and microvascular reconstruction. Scand J Plast Reconstr Surg Hand Surg. 2009; 43(2): I I3-I 16, doi: 10.1080/02844310701384066, indexed in Pubmed: 19308863.

5. Sekino $\mathrm{S}$, Takagi $\mathrm{H}$, Kato $\mathrm{T}$, et al. Nontraumatic pseudoaneurysm of the proximal ulnar artery with eosinophilia. J Vasc Surg. 2005; 42(6): 1233-1235, doi: 10.1016/j.jvs.2005.08.019, indexed in Pubmed: 16376222.

6. Wu G, Yang J, Zhang T, et al. The diagnostic value of non-contrast enhanced quiescent interval single shot (QISS) magnetic resonance angiography at $3 \mathrm{~T}$ for lower extremity peripheral arterial disease, in comparison to CT angiography. J Cardiovasc Magn Reson. 2016; I8(I): 71, doi: 10.1 186/s | 2968-016-0294-6, indexed in Pubmed: 27760564.

7. Buda SJ, Johanning JM. Brachial, radial, and ulnar arteries in the endovascular era: choice of intervention. Semin Vasc Surg. 2005; 18(4): 191-195, doi: 10.1053/j.semvascsurg.2005.09.004, indexed in Pubmed: 16360575.

8. Igari K, Kudo T, Toyofuku T, et al. Surgical treatment of aneurysms in the upper limbs. Ann Vasc Dis. 2013; 6(3): 637-64I, doi: 10.3400/avd.cr.13-00024, indexed in Pubmed: 24130621 . 